\title{
MYC maintains high-fidelity splicing
}

Overexpression of MYC affects many transcriptional networks, but which of these downstream pathways are crucial for MYC oncogenesis is not clear. A recent study suggests that MYC contributes to lymphomagenesis by upregulating transcription of key components involved in the assembly of core spliceosomal small nuclear ribonucleoprotein particles (snRNPs), therefore maintaining correct pre-mRNA splicing.

Koh and Bezzi et al. established that overexpression of MYC in a mouse model of lymphoma (E $\mu-M y c$ mice) upregulates transcription of components of the spliceosome,

\section{link MYC} directly to effects on the splicing machinery in particular snRNP assembly genes such as the arginine methyltransferase Prmt5. Overexpression was confirmed at the protein level and also observed in MYCoverexpressing human lymphoma samples. When $\mathrm{Prmt5}^{+/-}$mice (Prmt5 $5^{-1-}$ mice are embryonic lethal)

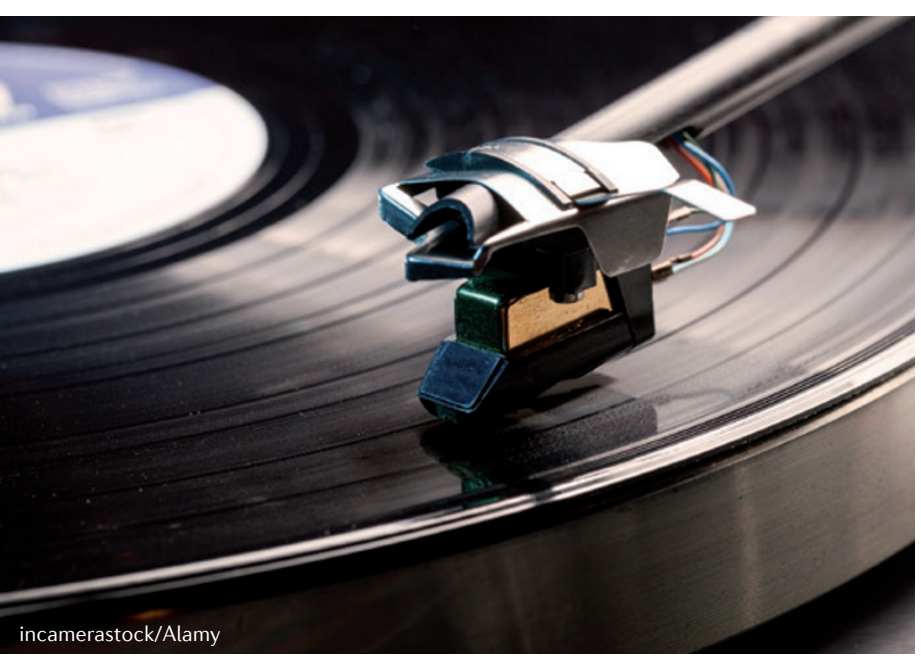

were crossed with $\mathrm{E} \mu-M y c$ mice, lymphoma development and disease burden were significantly reduced compared with $\mathrm{E} \mu-M y c$ controls. In addition, conditional deletion of Prmt5 in E $\mu-M y c$ lymphoma cells transplanted into mice indicated that PRMT5 is also important for tumour maintenance.

To better understand the role of PRMT5 in MYC-induced lymphomas, the authors conditionally deleted Prmt5 in pre-tumoural $\mathrm{B}$ cells isolated from $\mathrm{E} \mu-M y c$ mice; this increased apoptosis and cell cycle arrest, indicating that MYCinduced proliferation and survival requires PRMT5. RNA sequencing and analysis of transcript splicing in these cells indicated that Prmt5 deletion in the context of $\mathrm{E} \mu-\mathrm{M} y c$ expression altered the splicing of more than 150 transcripts, primarily resulting in aberrant splicing events with retained introns and skipped exons with weak $5^{\prime}$ donor sites. The authors examined 22 alternative splicing events further and all were confirmed to be increased in $\mathrm{E} \mu-M y c$ cells with a deletion of Prmt5, compared with cells that had either Prmt5 deletion or MYC overexpression alone. Many of these alternatively spliced mRNAs were predicted to be targets for nonsense-mediated mRNA decay or to be out of frame; indeed, in seven cases tested, the levels of protein translated from those mRNAs were reduced. Similar results to those seen in pre-tumoural B cells were observed when Prmt5 was conditionally deleted from $\mathrm{E} \mu-M y c$ lymphoma cells or when another core component of the splicing machinery $(\mathrm{SmB})$ was depleted, indicating that the effects of PRMT5 on splicing are indeed crucial for the observed phenotype.

The authors looked more closely at three splicing events altered by PRMT5 loss that might directly affect lymphomagenesis: that of ataxia telangiectasia and Rad3-related (Atr; skipped exon); E1A-binding protein p400 (Ep400; skipped exon); and dishevelled ( $D v l 1$; retained intron). Specific induction of each alternative splicing event using antisense oligonucleotides in $\mathrm{E} \mu-M y c \mathrm{~B}$ cells was sufficient to increase cell cycle arrest and apoptosis; however, it seems likely that a combination of events contributes to lymphoma reduction in vivo.

MYC has previously been associated with splicing, but these data now link MYC directly to effects on the splicing machinery. The authors also showed that complete deletion of Prmt5 at various stages of mouse development causes severe adverse effects that often lead to death. Therefore, although their data suggest that there might be a therapeutic window for targeting PRMT5 in tumours overexpressing MYC and PRMT5, any attempt to inhibit this pathway clinically would require the utmost caution.

Sarah Seton-Rogers

ORIGINAL RESEARCH PAPER Koh, C. M. \& Bezzi, M. et al. MYC regulates the core pre-mRNA splicing machinery as an essential step in lymphomagenesis. Nature http://dx.doi. org/10.1038/nature14351 (2015) 